\title{
ELECTRICALLY OPERATED STAIR LIFT FOR DIFFERENTLY ABLED PERSON
}

\author{
KEERTHANA D ${ }^{1}$, CB.SWATTHY ${ }^{2} \&$ SM.PARANTHAAMAN ${ }^{3}$ \\ ${ }^{1}$ Faculty of Mechatronics Engineering, Rajalakshmi Engineering College, Thandalam, Chennai, India \\ ${ }^{2,3}$ Students of Mechatronics Engineering, Rajalakshmi Engineering College, Thandalam, Chennai, India
}

\begin{abstract}
In this fast-growing world with huge technologies and resources, the need for precise control of wheelchair over stairs is not so far into successful existence. Not all the wheelchair proposed are portable and handy to operate. This journal aims to present a wheelchair which could climb over the stairs. This describes the electrically operated wheelchair-using battery; it can climb upon stairs by the help of the belt drive placed at the rear side of the chair model. It includes a switch for forwarding, backward, starts and stop. The chair set up is already been made attached along with the drive belt and it tends to be portable and easy in handling, as the start button is pressed the battery-driven belt starts to rotate along forwarding direction when the forward button is pressed and the same happens when the chair is brought down with the reverse button. This overall setup is made portable and handy in operation when compared with other models.

KEYWORDS: Smart Wheelchair, Electrically Operated, Belt Driven, Portable and Handy
\end{abstract}

Received: Jun 08, 2020; Accepted: Jun 28, 2020; Published: Sep 16, 2020; Paper Id.: IJMPERDJUN20201268

\section{INTRODUCTION}

As there is numerous growth in innovations been proposed in today's life, the introduction of the automatically operated wheelchair by sensors like sniff and puff sensor, accelerometer sensor, hand-held control have made disabled people to easily move along places, apart from all these the problem that is caused to move along the stair is still difficult and dangerous. As per the research done in 2001 it has been revealed that over 21 million Indians are suffering from anyone kind of disability. This, in turn, is equivalent to literally $2.1 \%$ of the whole population. Among those disabled in the country, 12.6 million are males and 9.3 million are females. The disability rate (number of disabled per, 100,000 populations) for the country as a whole works out to be 2130. This is 2,369 with males and 1,874 with females. The proportions are movement (27.9\%), speech (7.5\%), and hearing (5.8\%).As a whole, these people with such difficulties find it so hard to live their normal day to day life and thus came the automatically controlled wheelchair which tends to be at its best in its floor level movements. But when it comes with staircase climbing the difficulty is still prevailing, a method proposed earlier was the platform inclined lifts, which is a mechanical device of chair attached to the rail, this method had the difficulty in its huge setup and operation, this set up is not portable along with all places.

Passenger platform lifts are contained within a shat. The lift shaft has guide rails along two corners to guide the carrier, which is generally an L shaped platform attached to a mast. When using an open platform lift, the walls remain in the same place as the platform raises or lowers[1].There are two types of passenger platform lift, including enclosed cabin lifts. Cabin platform lifts work in the same way but rather than having an L shaped carrier, the platform is contained within a box to give the feel of a conventional lift[1]. Operation inside an open platform lift is by continuous pressure, and outside using a single touch button to call the lift to the floor[1]. For enclosed 
cabin lift, operation inside the cabin is a single touch. The next introduced was the star configured wheels along with the rotating wheel at the centre, this is manually operated by a person behind for moving forward and backward movements, this is easy when compared to the previous platform lift but involves a person guided along to push heavily along its direction, this method does not involve any electrically driven feature but involves heavy strength in moving up and down. It generally has multiple wheels which tend to move along in a triangular fashion one after the other. When the end-user provides the required motive power, the triangular configuration of the wheels makes it far easier to transform forward motion into upward motion according to its rotation. The report of this technique reported that it has uncounted problems during descending of the wheelchair in stairs, with some vibration problem been encountered.

The proposed work is with battery-operated drive belt to move along forward and backward direction which involves start/stop button with forwarding and backwards driven button, unless like other wheel type techniques this technique does not involve wheels but has a drive belt. This method is much easy in operation as it does not require much strength in moving the person along in the required direction. It has to be charged regularly. When compared this is so easy to take along with places and in operation. A single switch tends to move along both forward and backward control. During the forward control the front wheel of the wheelchair is locked as it does not need to work and in the same way while in backward control the back wheel of the wheelchair is made locked and according to the size of the staircase the drive belt is adjusted along with the required height by the adjustable holes along the sides. This technique is so easy in operation and portable.

To solve the difficulty faced by a disabled person in climbing up the stairs, a battery-operated mechanism to make the drive belt to move both in forwarding and backward directions along with several buttons in controlling its operation. Once the start button is pressed, the angle of the drive could be adjusted to perfectly suit up all types of stairs for its forward and backward movement. The forward and backward button tends the drive to move both up and down accordingly, as to move up in the stair the chair is placed in such a way that its drive belt is made to be locked up in an angle that suits the staircase. The adjustments at its side help to perfectly align the drive belt in an angle according to the stairs. The chair with lifting mechanism is suitable to be carried along to places and does not involve huge efforts in operation, both portable and handy in operation when compared with the earlier introduced techniques.

\section{LITERATURE REVIEW}

The main problem has been faced is the difficulty in making the chair, really portable in all aspects of places like making it possible to climb stairs and making it so comfortable for the patients without experiencing any sort of friction on the surface.

- Murray John Lawn (2002) et.al. Made a detailed study on all the types of wheelchair being introduced under the topic "Study of stair climbing assistive mechanisms for the disabled". It includes right from an old method like fixed chair stair-lift, platform stair-lift, scala mobile (which has a long piece of rod with a wheel attached below which can carry the whole chair when placed upon the surface), cluster wheel, dual wheel cluster. His work focuses on the study of stair-climbing capable mechanisms for the elderly or disabled. It includes common mobility assistive techniques and devices and recent advances in curb and stair climbing devices.

- Lippincott Williams \& Wilkins (2003) proposed the method of double wheel arrangement, under the topic "stairclimbing wheelchair approved" (journal of clinical engineering).Their concept is that the basic framework of the 
wheelchair is similar to that of the ordinary ones and it differs in a way that it tends to have a double wheel arrangement at both ends of the rear wheel, by this double wheel arrangement the person could climb up the stairs.

- Murray J Lawn (2003) et.al. Proposed the idea related to that of the triangular arrangement, under the topic "Modelling of a stair-climbing wheelchair mechanism with high single-step capability" (IEEE transactions on neural systems and rehabilitation engineering). In the field of providing mobility for the elderly and disabled the aspect of dealing with stairs continues largely unresolved. This paper focuses on presenting the development of a stair-climbing mechanism with high single step capability. This is based on front and rear wheel clusters which are connected to the base that is the chair via powered linkages so that it could permit both autonomous stair ascending and descending in the forward direction, and high single-step functionality for such as direct entry to and from a van.

\section{Inference from Literature Review}

As explained there are several mechanisms for making the chair accessible upon stairs, the main problem is dealing with its structure. The frame of the chair should be designed in such a way it should be portable and handy in operation. The frame is made to be easily folded into two half and so it tends to be easily portable along with places as required. The usage of the brushed DC motor causes the wear and tear of brushes along the passage of time and hence brushless DC motor is very much suitable. In cluster wheel arrangement the regular motion of different wheels causes uneven and discomfort during operation as it causes vibrations to take place and hence it is preferred to be of a single wheel or a long belt mechanism which is also quite easy and portable in operation is required.

\section{PROPOSED SYSTEM}

In platform inclined lift, its construction and its operation are huge and little difficult. It could not be constructed in all places and it could not be taken along while travelling along with places. Once mechanical error been noticed the whole set up should be made rechecked up. Instar wheel configuration along with the rotating hub at its centre, it is hard to move the disabled person along the direction. It does not involve any electrically driven methods and hence friction could occur when moving along the backward direction or during moving down the stairs. As a whole, it does not possess a smooth operation and does not provide comfort in movement. The triangular wheel arrangement technique stated that it had a problem during descending of the wheelchair in stairs. It had a smooth journey while ascending but while coming down from the steps, it was found that it experienced some vibration problems. Both the process is a little hard and not easy when travelling along with places.

\section{Objective}

Make the person stay in a comfortable state during the movements, easy in operation and make it much portable and handy in operation. Single switch operated mechanism for both the forward and backward direction. Much effort is not needed in moving the person along places as it is electrically operated technique. Could be easily made to be carried along with places during travelling. Does not cause discomforts during movements with adjustable setup. An electrically operated system and hence involves regular charging for efficient operation. 


\section{Proposed Method}

Battery operated drive belt to move along forward and backward direction. It involves start/stop button with forward and backwards driven button, this is much easy in operation as it does not requires much strength in moving the person along in the required direction. It has to be changed regularly for efficient processing. When compared this is so easy in portable along with places and in operation. A single switch tends to move along both forward and backward control. During the forward control, the front wheel of the wheelchair is locked as it does not need to work and in the same way while in backward control the back wheel of the wheelchair is made locked. According to the size of the staircase, the drive belt is adjusted along with the required height by the adjustable holes along the sides. This technique is so easy in operation and portable.

\section{BLOCK DIAGRAM}

Frame Work Design to discuss the design, it falls into the basic design (the design already exist) and the optimization design (changes been bought). The mentioned framework explains the complete model of the work. The basic design states the already existing frame setup of the wheelchair design. The wheel arrangement design is also already existing one. The optimization states the newly in co-operated ideas with the basic ideas, the belt placed at the backside of the chair helps in climbing up and down the stairs. Scaffolding caster wheels help in locking up the chair while in operation (when moving up and down the stairs).

Ergonomic design involves the use of self-tapping screws for folding and opening the arm and head side rest when required. The frame is designed in such a way that it could completely be folded up into two half. The working shows up the connection to make the wheelchair work up. As the switch is turned ON, the current flows from the battery to the drive belt via the chain and sprocket, shaft and roller to make the motor to rotate which makes the drive belt to rotate accordingly.

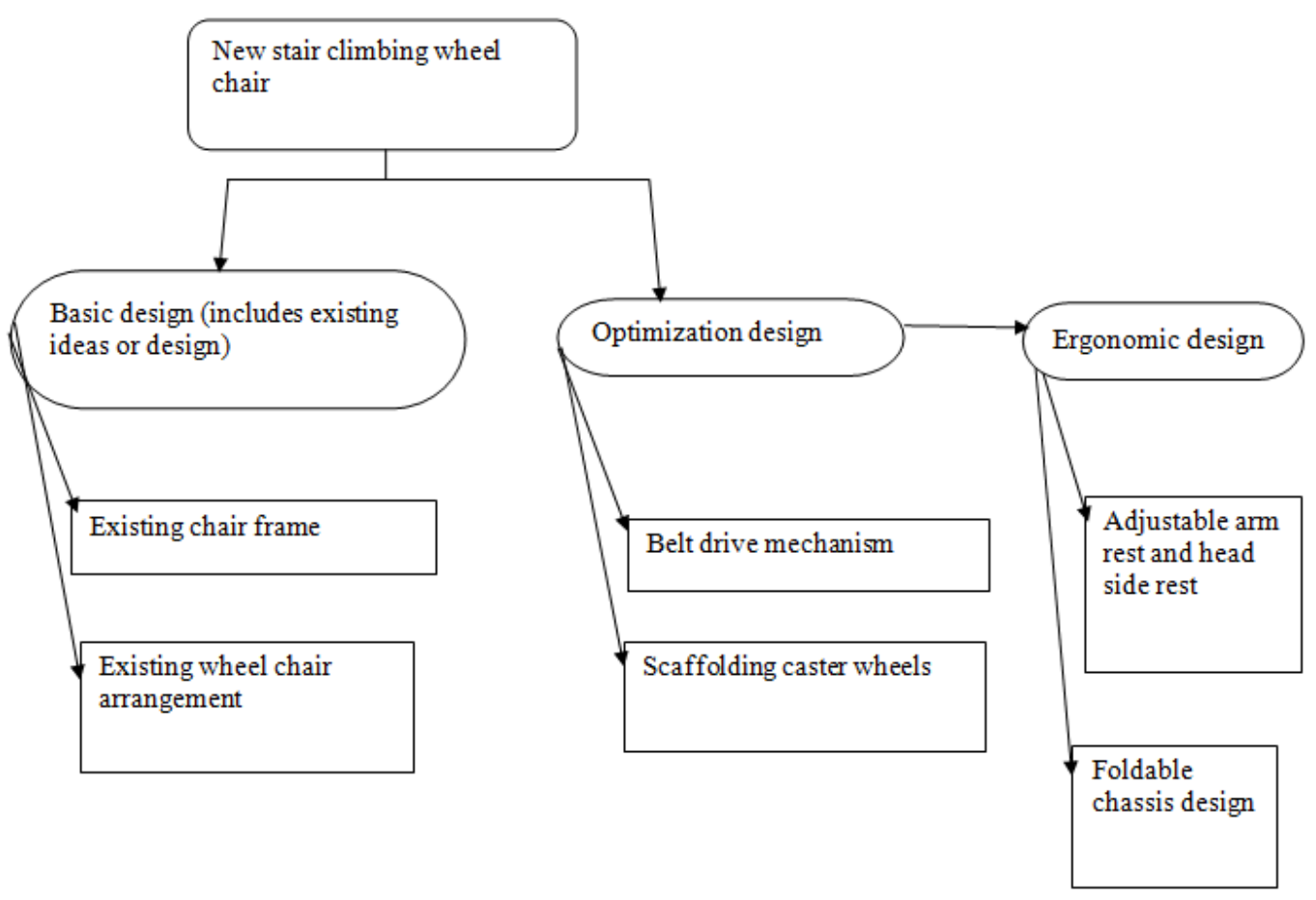

Figure 1: Design Format. 


\section{WORKING FLOW}

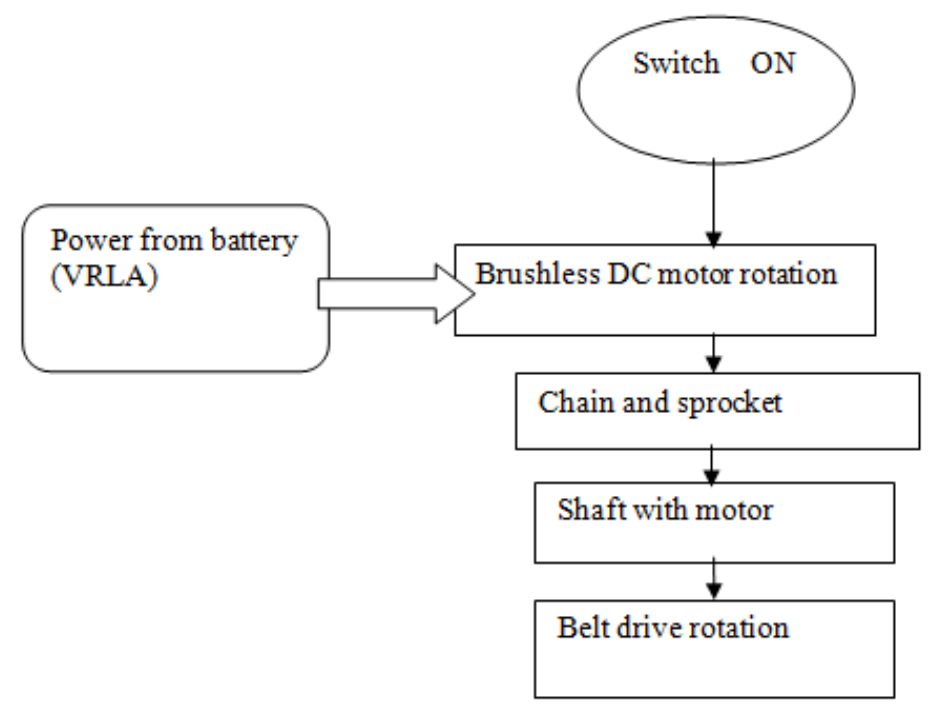

Figure 2: Working Format.

\section{METHODOLOGY}

\section{Construction}

It consists of the stainless steel frame; the design is similar to that of the normal frame of a chair with wood being mounted at the resting area. The handles at the side are welded in a way it can fold up if not required. Self-tapping screws are used to lower and raise the handle at the headrest side of the chair, the screws support in adjusting the frame outline. At the midportion of the chair, it is welded in a way that it makes the frame to be folded up into equal parts if the shape of inverted $\mathrm{V}$. The wheel at the bottom of the chair helps to move with load acting upon it. The front two wheels are of casting wheels and the rear end two scaffolding wheels provide good support in locking up the chair at an angle if required. The belt drive at the back is placed in an inclined angle when the chair is held normal to the surface the belt drive is inclined to the surface as shown in the figure.

\section{Working}

The working is generally using the drive which is placed at the back of the frame. The angle of the drive could be adjusted to perfectly suit up all types of stairs for its forward and backward movement. The forward and backward button tends the drive to move both up and down accordingly, as to move up in the stair the chair is placed in such a way that its drive belt is made to be locked up in an angle that suits the staircase. The adjustments at its side help to perfectly align the drive belt in an angle according to the stairs. The motor powered by the battery-operated the drive to move in forward and backward directions. As per the requirement, the chair is placed at an angle inclined upon the chair and it is made to move forward by the action of the drive rotating in a clockwise direction and while bringing down the drive rotates in an anticlockwise direction. This makes the chair to move in stair both forward and backwards.

\section{Simulation}

For maintaining the safety and stability of the chair and for solving the task of climbing upstairs and downstairs without any vibrations, simulation and analysis play the most important in our design. To know the design of our chair improves the property of the wheelchair or not the following reasons are needed to be satisfied. 
- The frame should be designed in such a way that it should be able to resist the expected loads under the static condition with the construction of a sufficiently large support base.

- The chassis should be strong enough to withstand a load of an average person.

- The scaffolding wheels should be strong and capable of locking a wheelchair when climbing up and down the stairs without causing any sort of difficulty.4. The overall structure should be examined using solid works and the output should be proper during operation without any disturbance.

\section{RESULT}

As by the overall working of the project, the structure is made as per the calculations and hence the operation is smooth and it does not cause any vibrations during its operation. The efficiency and the performance are as expected and it does a great job in its operation. The material selected doesn't get affected easily by heat or electricity; this tends to be an added advantage. The inclined angle of the belt is determined and fixed as per the calculations. This angle of the belt helps in all types of stairs within its limitations as specified before. Thus the overall performance and efficiency are obtained as expected and it helps the person in climbing up and down the stairs easily. In this work, the proposed idea is a new kind of stair-climbing wheelchair, which has a compact structure. It is easy in operation and also portable in construction. It could cope with flat or inclined terrain stairs within the limits as explained before. Thus the modelling of the frame and the complete structure is done in solid works and it is made to run to completely analyse the structure for different loads as possible. Upon several references from different journals the correct structure of the stairs and the required angle for the belt to be inclined is known. Based on the calculations, various components have been selected and used as per the correct requirement. The chair in operation tends to be smooth and handling of the chair is simple and overall the structure of the chair is designed in a much portable way and this is an added strength.

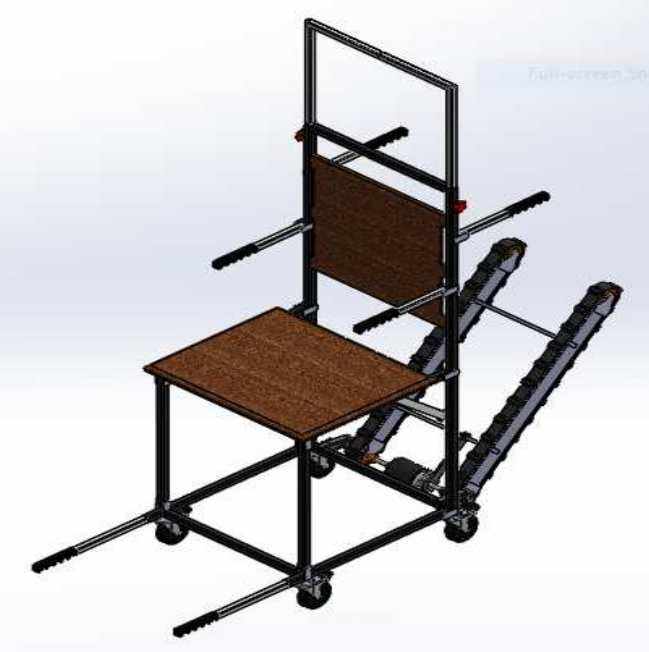

Figure 3: Solidworks Model. 


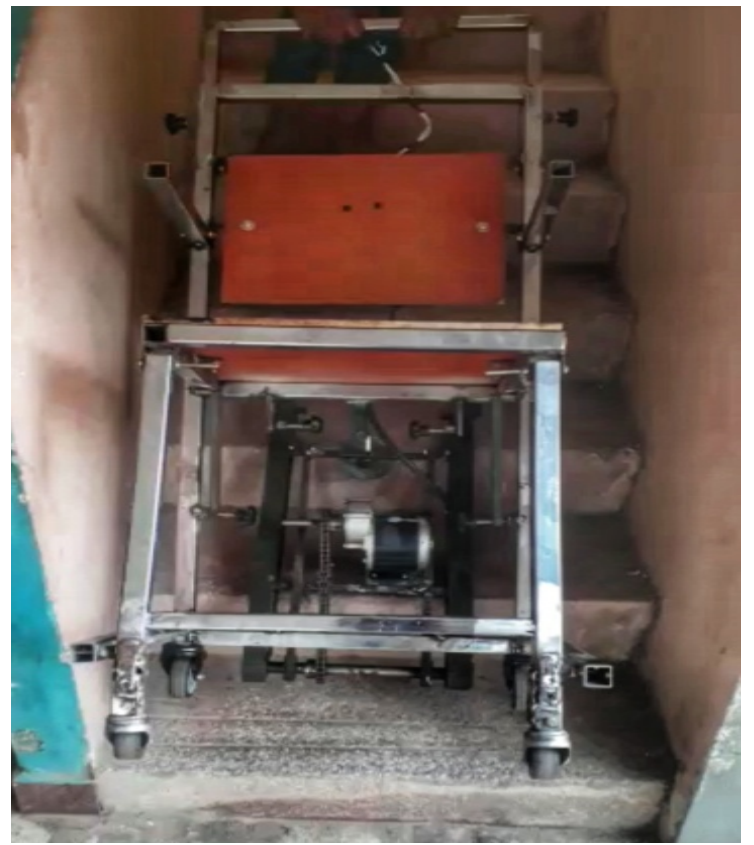

Figure 4: Output Model.

\section{FUTURE SCOPE}

Some improvements need to be done in the future, as mentioned below:

- Making up a small prototype and completely investigating the model to be assessable in all types of stairs and terrain and even upon big obstacles as possible.

- In replace for a battery that is to be always charged upon regular intervals even AC supply could also be used to run the motor.

- Improved usage of the locking system of the wheels could be appreciated.

- Going up and down the stairs without any assistance.

- Develop intelligent control to make it more automated.

- Sensor detection and alarm system can be installed which is used to notify the user when the wheelchair comes across obstacles

\section{REFERENCES}

1. R.A. Cooper, M.L. Boninger, R. Cooper and A. Kelleher, (2006), "Use of the independence 3000 IBOT transporter at home and in the community: A case report", Disability and Rehabilitation: Assistive Technology 1-2.

2. D. Ding and R.A. Cooper, (2005), "Electric powered wheelchairs, a review of current technology and insight into future directions", IEEE Control Systems Magazine 25.

3. Gaikwad, Rakesh Sayaji, and Girish Kamal Gautam. "Image Processing Based Colour Pointer to Drive Wheelchair Using Open CV."International Journal of Electronics, Communication \& Instrumentation Engineering Research and Development (IJECIERD) 5. 3, Jun 2015, 19-26 
4. N.M.A. Ghani, A.N.K. Nasir and M.O. Tokhi,(2014), "Optimization of fuzzy logic scaling parameters with a spiral dynamic algorithm in controlling a stair-climbing wheelchair: Ascending task”, proceeding of 19th International Conference on Methods and Models in Automation and Robotics (MMAR).

5. L.F.S.Coletta, E.R.Hruschka, A.Acharya and J. Ghosh, "Using metaheuristics to optimize the combination of classifier and cluster ensembles", Integrated Computer-Aided Engineering.

6. A.P. Engelbrecht,(2007), “Computational intelligence: An introduction”, Chichester, England, John Wiley and Sons.

7. Chandrasekhar, Gerald. "Design and Fabrication of a Low-Cost Assistive Device to Support the Basic Movements for Elderly People. International Journal of Mechanical and Production Engineering Research and Development (IJMPERD) 9.5, Oct 2019, 851-860

8. B. Fariba, R. Robert, J.M. Parviz and S. Gunther,(2000), "Biomechanical analysis of sit-to-stand transfer in healthy and paraplegic subjects", Clinical Biomechanics 15.

9. K.M. Goher,(2013), "Modelling and simulation of a reconfigurable wheelchair with sit-to-stand facility for a disabled child", Proceeding of IEEE 18Th International Conference on Methods and Models in Automation and Robotics, (MMAR).

10. Gaal RP, Rebholtz, N, Hotchkiss RD, Pfaelzer PF,(1997), "Wheelchair rider injuries: Causes and consequences for wheelchair design and selection", J Rehabil Res Dev.

11. Ummat S, Kirby RL,(1994), "Nonfatal wheelchair-related accidents reported to the National Electronic Injury Surveillance System”, Am J Phys Med Rehabil.

12. Nelson AL, Groer S, Palacios P, et al,(2010), "Wheelchair-related falls in veterans with spinal cord injury residing in the community: A prospective cohort study", Arch Phys Med Rehabil.

13. Chen W-Y, Jang Y, Wang J-D, et al., (2011), “Wheelchair-related accidents: Relationship with wheelchair-using behaviour in active community wheelchair users", Arch Phys Med Rehabil. 